\title{
Coherent control of the Goos-Hänchen shift
}

\author{
Ziauddin, ${ }^{1}$ Sajid Qamar, ${ }^{1}$ and M. Suhail Zubairy ${ }^{1,2}$ \\ ${ }^{1}$ Centre for Quantum Physics, COMSATS Institute of Information Technology, Islamabad, Pakistan \\ ${ }^{2}$ Institute for Quantum Studies and Department of Physics, Texas A\&M University, College Station, Texas 77843-4242, USA
}

(Received 31 July 2009; published 22 February 2010)

\begin{abstract}
The behavior of the Goos-Hänchen $(\mathrm{GH})$ shifts in the reflected and transmitted light beam which is incident on a cavity containing an intracavity medium of three-level or four-level atoms with electromagnetically induced transparency (EIT) is discussed. We report a coherent control of the GH shift in a fixed configuration or device via superluminal and subluminal wave propagation. For superluminal wave propagation, we observe negative GH shifts in the reflected part of the incident light whereas the shifts are positive in the transmitted light beam. This corresponds to the negative group index of the cavity in the former case and positive group index of the cavity in the latter. For subluminal wave propagation, the behavior of the GH shifts in the reflected light changes and positive shifts appear; however, the GH shifts in the transmitted light remains positive. The corresponding group index of the cavity is positive in both cases.
\end{abstract}

DOI: 10.1103/PhysRevA.81.023821

PACS number(s): 42.25.Bs, 42.50.Gy

\section{INTRODUCTION}

In 1947, Goos and Hänchen [1] experimentally observed evidence of the displacement of a light beam when it is totally reflected at a dielectric interface. This displacement, called the Goos-Hänchen shift (GH shift), is perpendicular to the direction of propagation of the reflected beam in the plane of incidence. Since then several theories have been developed and a large amount of literature is now available to explain the positive GH shift. These include, for example, total internal reflection when energy conservation has been imposed [2], reflection and refraction of light at a plane interface [3], and lateral displacement of light in the multilayered and periodic structures [4]. Besides, a negative GH shift may appear in negative permitivity media [4], absorbing media [5-9], and negative refractive media [10]. The negative GH shift has also been noticed in the transmitted beam [11] when it passes through a slab of an optically denser dielectric medium. It has also been observed that depending on the thickness of the left-handed slab the GH shift of the transmitted beam can be negative as well as positive when a light beam passes through the slab [12]. However, in all these proposals, the manipulation of the GH shift cannot be done for a fixed configuration of the medium. The GH shift has some interesting applications in optical sensing [13], where it can be used to measure the beam angle, refractive index, and displacement, for surface and film thickness studies [14], further, it can probe irregularities, roughness, etc. on the surface of an isotropic spatially dispersive medium [7]. Therefore, any scheme which deals in control and manipulation of the GH shift using a fixed configuration or device seems to be more appropriate.

In some earlier studies, it has been observed that the susceptibility of the intracavity medium can be changed by using a coherent driving field [15-17]. On the basis of these investigations, very recently, a scheme has been suggested for control of the Goos-Hänchen shift by modifying the susceptibility of a two-level atomic medium in a fixed cavity [18]. In this scheme, it has been proposed that the positive as well as negative GH shift can be observed in the reflected and transmitted probe light beams, without changing the structure of the cavity, by adjusting the intensity and detuning of the coherent driving field. The noticeable point, however, is that a large off-resonant interaction of the probe light beam with the corresponding atomic transition has been considered in this scheme. This is to avoid strong absorption of the light inside the cavity when the probe light beam is resonant with the corresponding atomic transition. The idea is in general based on some earlier work on the manipulation of the refractive index and absorption in the fixed medium [19] and more specifically when the refractive index of a two-level atomic medium is enhanced with less absorption of the probe light beam [20].

Superluminal or subluminal wave propagation in an atomic medium corresponds to the negative or positive group index of the medium, respectively, and a single knob is sufficient to control it via electromagnetically induced transparency (EIT) [21]. The knob may be the intensity or the phase of the coherent driving field. The positive or negative GH shift in the reflected and transmitted light may correspond to the positive or negative refractive index of the medium, respectively; therefore, a relevant question arises as to whether one can manipulate the GH shift via superluminal and subluminal wave propagation using EIT. This may result in getting negative as well as positive GH shifts in the reflected and transmitted light without disturbing the configuration of the medium and for almost zero absorption of the light beam inside the medium.

In this proposal, we investigate this question considering a cavity with two walls of some dielectric material and an intracavity atomic medium while the light is incident on one of the walls of the cavity. The superluminal and subluminal propagation of light inside a three- or fourlevel atomic medium can be coherently controlled via the driving field Rabi frequency $[15,17,21]$. We expect a similar control of the GH shifts using the coherent driving fields when the light is reflected from or transmitted through the cavity. We consider three- and four-level atomic systems for the intracavity media which follow an EIT configuration as described in the earlier studies [15,17,21]. Superluminal or subluminal wave propagation inside these atomic media corresponds to the negative or positive group index of the media, respectively. However, the negative and positive GH 


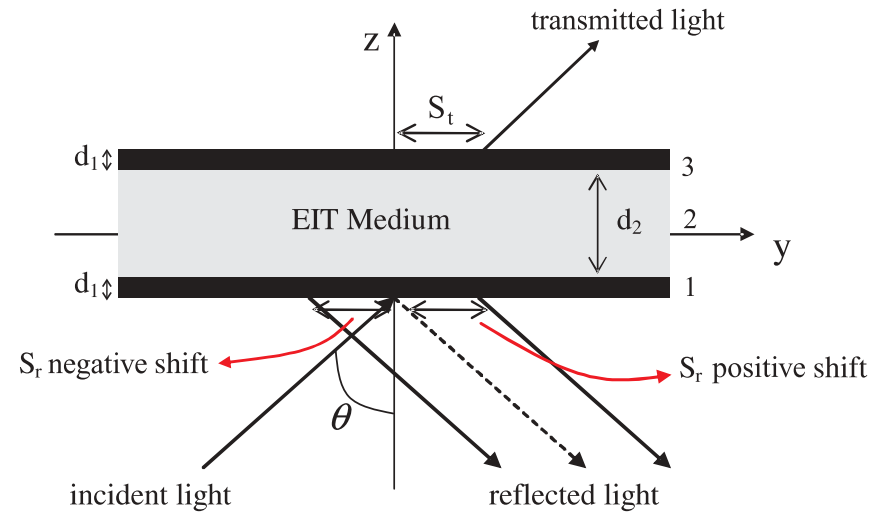

FIG. 1. (Color online) Schematic of the system. The walls of the cavity are of some nonmagnetic dielectric substance with width $d_{1}$ each and the length of the intracavity medium is $d_{2}$. The light is incident on the cavity wall at an angle $\theta$ along the $z$ axis, which is reflected and transmitted with positive or negative GH shift.

shifts in the reflected or transmitted light which is incident on one of the walls of the cavity correspond to the negative and positive group index of the cavity and not of the atomic medium only, which is the intracavity medium. The threelevel atomic system in EIT configuration undergoes a strong absorption during the superluminal propagation of the resonant probe light beam [15]. This problem can be resolved if the three-level atomic system is replaced with a four-level atomic system [21].

The organization of this article is as follows: In Sec. II, we present the model to find the GH shifts in the reflected and transmitted light beams incident on a cavity. Section III deals with the atom-field system for the intracavity medium; we consider three-level and four-level atomic media and present the expressions for the susceptibilities in each case. In Sec. IV, we discuss the results via numerical simulation and finally summarize our results and discussion.

\section{MODEL}

We consider a system where TE-polarized probe light beam $E_{p}$ having angular frequency $\omega_{p}$ is incident on a cavity from the vacuum with permitivity $\varepsilon_{0}=1$, as shown in Fig. 1 . The cavity is a stratified medium consisting of three layers, labelled 1,2 , and 3 in the schematics. Layers 1 and 3, which are acting as walls of the cavity, are nonmagnetic dielectric slabs with the same thickness $d_{1}$ and permitivity $\varepsilon_{1}$. Layer 2 is the intracavity medium with thickness $d_{2}$ and permitivity $\varepsilon_{2}$. The incident probe light beam makes an angle of $\theta$ with the $z$ axis and the intracavity medium can be gas of three-level or four-level atoms [22,23] and can be some semiconductor [24-29]. The strong coherent fields are applied to drive three- or four-level atomic systems in an EIT configuration, as described elsewhere $[15,21]$.

The incident probe light beam is reflected back or transmitted through the cavity with a lateral shift (GH shift). Given that the incident light beam has a large width (narrow angular spectrum, $\Delta k \ll k$ ), the lateral shift in the reflected or transmitted light can be calculated by using stationary phase theory $[11,30]$ as

$$
S_{r, t}=-\frac{\lambda}{2 \pi} \frac{d \varphi_{r, t}}{d \theta},
$$

where $\varphi_{r, t}$ is the phase associated with the reflection $r\left(k_{y}, \omega_{p}\right)$ or transmission $t\left(k_{y}, \omega_{p}\right)$ coefficients (with reflection and transmission coefficients being complex quantities). As a result the lateral or GH shift in the reflected and transmitted probe light beams can be expressed as [18]

$$
\begin{aligned}
S_{r}= & -\frac{\lambda}{2 \pi\left|r\left(k_{y}, \omega_{p}\right)\right|^{2}}\left\{\operatorname{Re}\left[r\left(k_{y}, \omega_{p}\right)\right] \frac{d \operatorname{Im}\left[r\left(k_{y}, \omega_{p}\right)\right]}{d \theta}\right. \\
& \left.-\operatorname{Im}\left[r\left(k_{y}, \omega_{p}\right)\right] \frac{d \operatorname{Re}\left[r\left(k_{y}, \omega_{p}\right)\right]}{d \theta}\right\}
\end{aligned}
$$

and

$$
\begin{aligned}
S_{t}= & -\frac{\lambda}{2 \pi\left|t\left(k_{y}, \omega_{p}\right)\right|^{2}}\left\{\operatorname{Re}\left[t\left(k_{y}, \omega_{p}\right)\right] \frac{d \operatorname{Im}\left[t\left(k_{y}, \omega_{p}\right)\right]}{d \theta}\right. \\
& \left.-\operatorname{Im}\left[t\left(k_{y}, \omega_{p}\right)\right] \frac{d \operatorname{Re}\left[t\left(k_{y}, \omega_{p}\right)\right]}{d \theta}\right\} .
\end{aligned}
$$

The reflection and transmission coefficients, which are required to find the GH shift [Eqs. (2) and (3)], can be calculated as follows [31].

The input and output of the electric field which is propagating through the stratified medium can be related to each other via a transfer matrix of the form [31,32]

$$
M_{j}\left(k_{y}, \omega_{p}, d_{j}\right)=\left(\begin{array}{lc}
\cos \left(k_{z}^{j} d_{j}\right) & i \sin \left(k_{z}^{j} d_{j}\right) / q_{j} \\
i q_{j} \sin \left(k_{z}^{j} d_{j}\right) & \cos \left(k_{z}^{j} d_{j}\right)
\end{array}\right),
$$

where $k_{z}^{j}=\sqrt{\varepsilon_{j} k^{2}-k_{y}^{2}}$ is the $z$ component of the wave number, $q_{j}=k_{z}^{j} / k, d_{j}$ is the thickness, and $j$ represents the $j$ th layer of the medium. As mentioned earlier, in the present scheme, there are three layers in the stratified medium. The total transfer matrix of the cavity is given by [18,31]

$$
Q\left(k_{y}, \omega_{p}\right)=M_{1}\left(k_{y}, \omega_{p}, d_{1}\right) M_{2}\left(k_{y}, \omega_{p}, d_{2}\right) M_{3}\left(k_{y}, \omega_{p}, d_{1}\right),
$$

where each $M_{1}\left(k_{y}, \omega_{p}, d_{1}\right), M_{2}\left(k_{y}, \omega_{p}, d_{2}\right)$, and $M_{3}\left(k_{y}, \omega_{p}\right.$, $\left.d_{1}\right)$ is a unimodular matrix. The matrix $Q\left(k_{y}, \omega_{P}\right)$ is therefore also unimodular.

We can calculate the reflection and transmission coefficients using the total transfer matrix (5) as

$r\left(k_{y}, \omega_{p}\right)=\frac{\cos \xi\left(q_{0}^{2}-q_{1}^{2}\right) q_{1} q_{2} \sin 2 \alpha+\left[q_{1}^{2}\left(q_{0}^{2}-q_{2}^{2}\right) \cos ^{2} \alpha+\left(q_{1}^{4}-q_{0}^{2} q_{2}^{2}\right) \sin ^{2} \alpha\right] \sin \xi}{q_{1} q_{2} \cos \xi\left[2 i q_{0} q_{1} \cos 2 \alpha+\left(q_{0}^{2}+q_{1}^{2}\right) \sin 2 \alpha\right]+\left[q_{1}^{2}\left(q_{0}^{2}+q_{2}^{2}\right) \cos ^{2} \alpha-\left(q_{1}^{4}+q_{0}^{2} q_{2}^{2}\right) \sin ^{2} \alpha-i q_{0} q_{1}\left(q_{1}^{2}+q_{2}^{2}\right) \sin 2 \alpha\right] \sin \xi}$ 
and

$t\left(k_{y}, \omega_{p}\right)=\frac{2 i q_{0} q_{1}^{2} q_{2}}{q_{1} q_{2} \cos \xi\left[2 i q_{0} q_{1} \cos 2 \alpha+\left(q_{0}^{2}+q_{1}^{2}\right) \sin 2 \alpha\right]+\left[q_{1}^{2}\left(q_{0}^{2}+q_{2}^{2}\right) \cos ^{2} \alpha-\left(q_{1}^{4}+q_{0}^{2} q_{2}^{2}\right) \sin ^{2} \alpha-i q_{0} q_{1}\left(q_{1}^{2}+q_{2}^{2}\right) \sin 2 \alpha\right] \sin \xi}$,

where $q_{0}=k_{z} / k$ (with $k_{z}$ the $z$ component of the wave number in vacuum), $k_{y}$ is the $y$ component of the wave number $k=$ $\omega_{p} / c$ in vacuum, $c$ is the velocity of light in vacuum, and

$$
\begin{aligned}
\alpha & =d_{1} k \sqrt{\varepsilon_{1}-\sin ^{2} \theta}, \\
\xi & =d_{2} k \sqrt{\varepsilon_{2}-\sin ^{2} \theta} .
\end{aligned}
$$

The permitivity $\varepsilon_{2}$ and the susceptibility $\chi$ of the intracavity medium can be related to each other via the relation [31]

$$
\varepsilon_{2}=1+\chi
$$

and $\chi$ can be written as [15]

$$
\chi=\chi^{\prime}+i \chi^{\prime \prime} \text {, }
$$

where $\chi^{\prime}$ and $\chi^{\prime \prime}$ be the real and imaginary parts of $\chi$, which give the dispersion and absorption properties of the intracavity medium, respectively. These quantities can be manipulated by using the coherent control of the strong driving fields to get superluminal or subluminal light propagation $[15,21,33]$.

Equations (6) and (7) along with expressions (8) and (9) show that the reflection and the transmission coefficients depend on the permitivities of the cavity walls and the intracavity medium, that is, $\varepsilon_{1}$ and $\varepsilon_{2}$, respectively. These coefficients also depend on the thickness of the cavity walls $\left(d_{1}\right)$, thickness of the intracavity medium $\left(d_{2}\right)$, and the incident angle of the probe light beam $(\theta)$. Further, using Eq. (10), we can observe that the reflection and transmission coefficients depend on the susceptibility $\chi$ of the intracavity medium. As a result, the GH shifts [Eqs. (2) and (3)] in the reflected and transmitted probe light beams also depend on all these parameters.

The group index of the medium is another relevant quantity which is related to the superluminal and subluminal behavior of the light propagation through the medium. The group index of the atomic medium inside the cavity can be calculated by using the real part of the susceptibility [21,33]. It is negative for superluminal wave propagation and positive for subluminal wave propagation. However, the behavior of the group index corresponding to the total cavity which includes the walls of the cavity and the atomic medium inside the cavity may be different from the group index of the intracacvity medium alone. Therefore, it is instructive to study the dependence of the GH shifts upon the group index of the cavity corresponding to superluminal and subluminal light propagation.

We consider that the cavity consists of three layers; layers 1 and 3 are the walls of the cavity with thickness $d_{1}$ each, and layer 2 is the intracavity medium with thickness $d_{2}$ (Fig. 1). Therefore, the total width or thickness of the cavity is $L=$ $2 d_{1}+d_{2}$. The group index of the cavity can be calculated by using the relation between the group velocity $v_{g}$ and the group delay $\tau_{g}$. The expression for the group velocity corresponding to the reflected or transmitted light beam is given by

$$
v_{g}^{r, t}=\frac{L}{\tau_{g}^{r, t}}
$$

where the superscripts $r, t$ correspond to reflection and transmission parts of the incident light beam. The corresponding group delay of the cavity can be written as [34]

$$
\tau_{g}^{r, t}=\frac{\partial \varphi_{r, t}}{\partial \omega_{p}}
$$

Furthermore, the group index, defined as the ratio of $c$ (the speed of light in vacuum) to the group velocity $v_{g}$, can be approximated as [34]

$$
N_{g}^{r, t}=c / v_{g}^{r, t} \approx \frac{1}{L} \frac{d \varphi_{r, t}}{d \omega_{p}} .
$$

This shows that the group index of the cavity $\left(N_{g}^{r, t}\right)$ depends on the thickness of the cavity and the derivative (with respect to probe light frequency) of the phase associated with the reflected or the transmitted probe light beam. We expect that the GH shift is positive or negative depending on whether the group index $N_{g}^{r, t}$ is positive or negative, respectively.

\section{ATOM-FIELD SYSTEM FOR THE INTRACAVITY MEDIUM}

As has been described in the previous section, we calculate the susceptibility of the intracavity medium, which is needed to find the $\mathrm{GH}$ shift in the reflected and transmitted light beams [Eqs. (2) and (3)]. In the following, we consider the interaction of three-level and four-level atoms (intracavity medium) with the corresponding driving and weak probe fields to calculate the dispersion-absorption relation (11).

\section{A. Three-level EIT configuration}

First, we consider a three-level atomic system with levels $|a\rangle,|b\rangle$, and $|c\rangle$ which is interacting with a coherent driving field of frequency $\omega_{d}$ and a weak TE-polarized probe light field of frequency $\omega_{p}$, as shown in Fig. 2(a). The driving field is resonant with the atomic transition $|a\rangle$ to $|c\rangle$ and the probe light field is incident on the cavity from the vacuum $\left(\varepsilon_{0}=1\right)$ at an angle $\theta$ in the $z$ direction. The probe field with a detuning $\Delta$ is applied to the atomic transition between levels $|a\rangle$ and $|b\rangle$. The corresponding Hamiltonian can be written as

$$
H=H_{0}+H_{I},
$$

where the free part $\left(H_{0}\right)$ and interaction part $\left(H_{I}\right)$ of the total Hamiltonian are

$$
H_{0}=\hbar \omega_{a}|a\rangle\left\langle a\left|+\hbar \omega_{b}\right| b\right\rangle\left\langle b\left|+\hbar \omega_{c}\right| c\right\rangle\langle c|,
$$




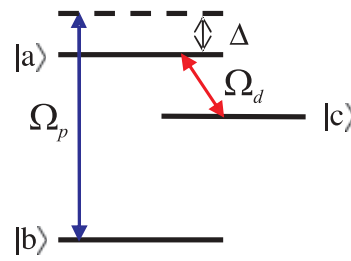

(a) (b)

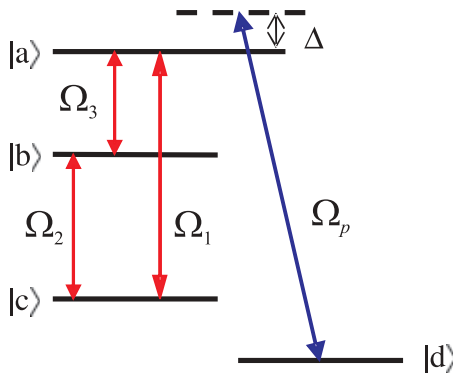

FIG. 2. (Color online) EIT configurations for (a) a three-level system and (b) a four-level system.

$$
H_{I}=-\frac{\hbar}{2}\left(\Omega_{d} e^{-i \omega_{d} t}|a\rangle\left\langle c\left|+\Omega_{p} e^{-i \omega_{p} t}\right| a\right\rangle\langle b|\right)+\text { H.c. },
$$

where $\Omega_{d}$ and $\Omega_{p}$ are the Rabi frequencies corresponding to the coherent driving field and weak probe field, respectively.

We use the master equation approach [15] and the relation $P=\chi\left(\omega_{p}\right) \epsilon_{0} E_{p}$, where $P$ is the polarization between atomic transition $|a\rangle$ and $|b\rangle$. The resulting expressions for the real and imaginary parts of the linear susceptibility $\chi\left(\omega_{p}\right)$ of the intracavity medium are [15]

$$
\chi^{\prime}\left(\omega_{p}\right)=\frac{4 \beta \Delta\left[\Omega_{d}^{2}-4\left(\gamma^{2}+\Delta^{2}\right)\right]}{16\left(\gamma^{2}+\Delta^{2}\right)\left(\Gamma^{2}+\Delta^{2}\right)+8\left(\gamma \Gamma-\Delta^{2}\right) \Omega_{d}^{2}+\Omega_{d}^{4}}
$$

and

$$
\chi^{\prime \prime}\left(\omega_{p}\right)=\frac{4 \beta\left[4 \Gamma\left(\gamma^{2}+\Delta^{2}\right)+\gamma \Omega_{d}^{2}\right]}{16\left(\gamma^{2}+\Delta^{2}\right)\left(\Gamma^{2}+\Delta^{2}\right)+8\left(\gamma \Gamma-\Delta^{2}\right) \Omega_{d}^{2}+\Omega_{d}^{4}},
$$

with $\gamma$ and $\Gamma$ being the atomic decay rates from $|c\rangle$ to $|b\rangle$ and $|a\rangle$ to $|b\rangle$, respectively, and the detuning parameter $\Delta=$ $\omega_{p}-\omega_{a b}$; details can be found elsewhere [15]. The parameter $\beta$ is defined as

$$
\beta=N_{a}\left|\wp_{a b}\right|^{2} /\left(\epsilon_{0} \hbar\right),
$$

where $\wp_{a b}$ is the dipole matrix element of the transition $|a\rangle \rightarrow$ $|b\rangle$ and $N_{a}$ is the atom number density.

\section{B. Four-level EIT configuration}

Second, we consider a four-level atomic system with energy levels $|a\rangle,|b\rangle,|c\rangle$, and $|d\rangle$. The atomic transitions $|a\rangle$ to $|c\rangle$ and $|b\rangle$ are resonant with two strong driving fields of frequencies $\omega_{1}$ and $\omega_{3}$, respectively, whereas the dipole forbidden transition from $|b\rangle$ to $|c\rangle$ is resonant with a third strong driving field of frequency $\omega_{2}$. The corresponding Rabi frequencies are $\Omega_{1}$, $\Omega_{3}$, and $\Omega_{2}$. The weak probe light beam of frequency $\omega_{p}$ is applied to the atomic transition $|a\rangle$ to $|d\rangle$ with detuning $\Delta$; the corresponding Rabi frequency is $\Omega_{p}$ [see Fig. 2(b)]. The Hamiltonian for the system is given by

$$
H=H_{0}+H_{I},
$$

where the free part $\left(H_{0}\right)$ and interaction part $\left(H_{I}\right)$ of the Hamiltonian $(H)$ can be written as

$H_{0}=\hbar \omega_{a}|a\rangle\left\langle a\left|+\hbar \omega_{b}\right| b\right\rangle\left\langle b\left|+\hbar \omega_{c}\right| c\right\rangle\left\langle c\left|+\hbar \omega_{d}\right| d\right\rangle\langle d|$

and

$$
\begin{aligned}
H_{I}= & -\frac{\hbar}{2}\left(\Omega_{1} e^{-i \omega_{1} t}|a\rangle\left\langle c\left|+\Omega_{2} e^{-i \omega_{2} t}\right| b\right\rangle\langle c|\right. \\
& \left.+\Omega_{3} e^{-i \omega_{3} t}|a\rangle\left\langle b\left|+\Omega_{p} e^{-i \omega_{p} t}\right| a\right\rangle\langle b|\right)+ \text { H.c. }
\end{aligned}
$$

Following a similar procedure, as has been described in [21], the real and imaginary parts of the linear susceptibility $\chi\left(\omega_{p}\right)$ can be written as [21]

$$
\begin{gathered}
\chi^{\prime}\left(\omega_{p}\right)=\frac{-2 \beta\left\{\gamma_{2}^{2}\left(4 \Delta^{3}-\Delta \Omega_{1}^{2}\right)+\left(4 \Delta^{2}-\Omega_{2}^{2}\right)\left[4 \Delta^{3}+\Omega_{1} \Omega_{2} \Omega_{3}-\Delta\left(\Omega_{1}^{2}+\Omega_{2}^{2}+\Omega_{3}^{2}\right)\right]\right\}}{A}, \\
\chi^{\prime \prime}\left(\omega_{p}\right)=\frac{\beta\left\{\gamma_{1}\left[4 \gamma_{2}^{2} \Delta^{2}+\left(\Omega_{2}^{2}-4 \Delta^{2}\right)^{2}\right]+\gamma_{2}\left(\Omega_{1} \Omega_{2}-2 \Delta \Omega_{3}\right)^{2}\right\}}{A},
\end{gathered}
$$

where

$$
\begin{aligned}
A= & \gamma_{2}^{2}\left[4 \gamma_{1}^{2} \Delta^{2}+\left(\Omega_{1}^{2}-4 \Delta^{2}\right)^{2}\right]+\gamma_{1}^{2}\left(\Omega_{2}^{2}-4 \Delta^{2}\right)^{2} \\
& +2 \gamma_{1} \gamma_{2}\left(\Omega_{1} \Omega_{2}-2 \Delta \Omega_{3}\right)^{2}+4\left[4 \Delta^{3}+\Omega_{1} \Omega_{2} \Omega_{3}\right. \\
& \left.-\Delta\left(\Omega_{1}^{2}+\Omega_{2}^{2}+\Omega_{3}^{2}\right)\right]^{2},
\end{aligned}
$$

where $\gamma_{1}$ and $\gamma_{2}$ being the atomic decay rates from $|a\rangle$ and $|b\rangle$, respectively, to $|d\rangle$ and the detuning parameter $\Delta=\omega_{p}-\omega_{a d}$. The parameter $\beta$ is defined as

$$
\beta=2 N_{a}\left|\wp_{a d}\right|^{2} /\left(\epsilon_{0} \hbar\right) .
$$

In reaching Eqs. (24)-(26), we also consider that the collective phase $\phi=\phi_{2}+\phi_{3}-\phi_{1}=0$, where phases $\phi_{1}, \phi_{2}$, and $\phi_{3}$ correspond to the three driving field Rabi frequencies $\Omega_{1}, \Omega_{2}$, and $\Omega_{3}$, respectively.

\section{RESULTS AND DISCUSSION}

We discuss the behavior of the GH shift in the reflected and transmitted probe light beams, which are incident on the cavity, for superluminal and subluminal wave propagation. We consider that the intracavity medium consists of three-level atoms with the atom-field configuration as shown in Fig. 2(a). We recall that, for nonzero driving field $\left(\Omega_{d} \neq 0\right)$, the intracavity medium becomes transparent for the probe light beam at resonance $(\Delta=0)$ [15]. This is well known as EIT. The dispersion properties of the three-level EIT medium exhibit slow group velocity of the probe light beam. However, this subluminal behavior becomes superluminal, accompanying 

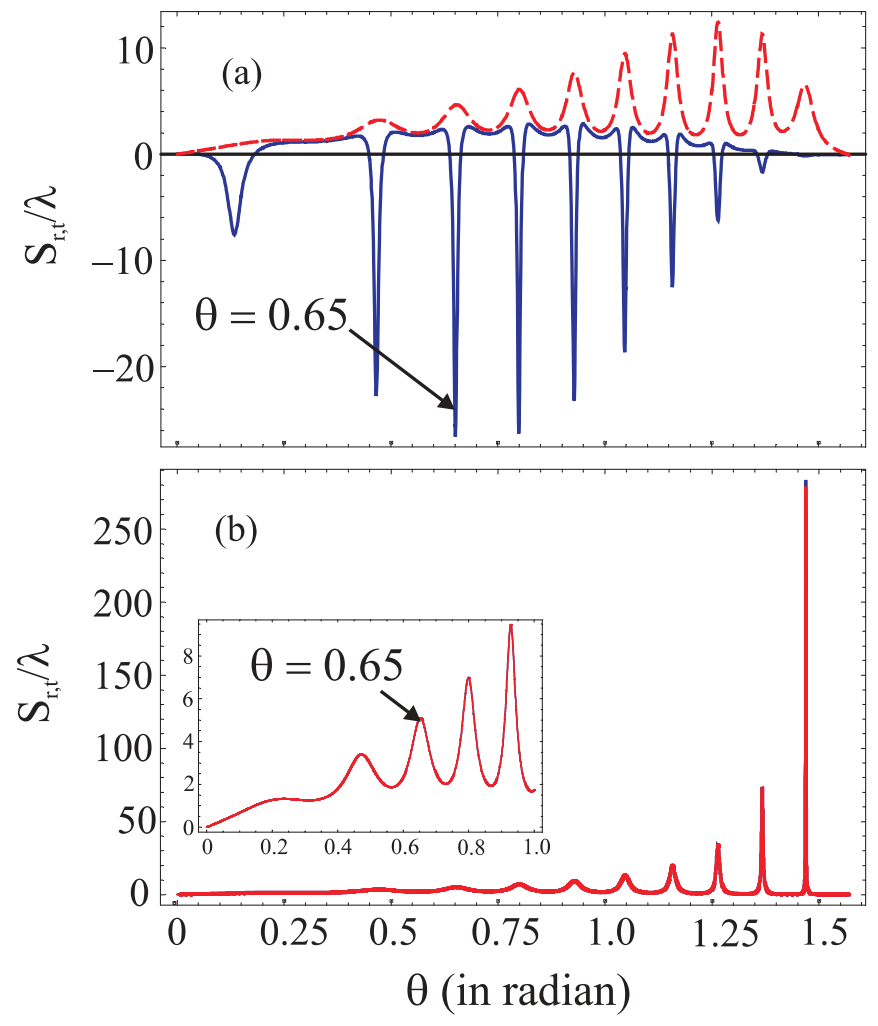

FIG. 3. (Color online) Three-level scheme showing GH shift behavior for different incident angles ( 0 to $\pi / 2 \mathrm{rad}$ ) of the probe light beam: (a) $\Omega_{d}=0$; reflected beam (solid line) and transmitted beam (dotted line). (b) $\Omega_{d}=5 \Gamma$; exactly similar behavior for reflected and transmitted beams is observed; the inset shows the enlarged view for $\theta=0$ to $1 \mathrm{rad}$. The other parameters are $\varepsilon_{1}=2.22, \Gamma=1 \mathrm{MHz}$, $\gamma=10^{-7} \Gamma, \beta=5 \times 10^{-3} \Gamma, \omega_{a b} /(2 \pi)=300 \mathrm{THz}, d_{1}=0.2 \mu \mathrm{m}$, $d_{2}=5 \mu \mathrm{m}$, and $\Delta=0$.

strong absorption of the probe light beam when the driving field is switched off $\left(\Omega_{d}=0\right)$.

As described earlier, we consider $N_{g}$ as the group index of the total cavity, which includes the two walls of the cavity having thickness $d_{1}$ each and the intracavity medium between these two walls with thickness $d_{2}$. We expect that the lateral shift or Goos-Hänchen shift is positive when $N_{g}$ is positive and it is negative when $N_{g}$ is negative for subluminal and superluminal light propagation, respectively. The controlling parameter for switching from negative to positive GH shift and vice versa is the intensity of the coherent driving field. We note that the required analytical expressions for the GH shift $\left(S_{r, t}\right)$ are rather complicated; therefore, in the following we study the behavior of the GH shifts in the reflected and transmitted light numerically.

The suitable choice of parameters here are $\beta=5 \times 10^{-3} \Gamma$, $\varepsilon_{1}=2.22, \Gamma=1 \mathrm{MHz}, \gamma=10^{-7} \Gamma, \omega_{a b} /(2 \pi)=300 \mathrm{THz}$, $d_{1}=0.2 \mu \mathrm{m}$, and $d_{2}=5 \mu \mathrm{m}$, which are in line with earlier work [18]. We also consider that the probe field is resonant with the atomic transition $|a\rangle$ to $|b\rangle$ (i.e., $\Delta=0$ ). In Fig. 3, we plot behavior of the GH shifts versus incident angle of the probe light beam ranging from 0 to $\pi / 2 \mathrm{rad}$. We choose two different values of the driving field Rabi frequency $\Omega_{d}$ : (i) $\Omega_{d}=0$ and (ii) $\Omega_{d}=5 \Gamma$. The Rabi frequency $\Omega_{d}=0$ corresponds to the case when superluminal propagation of the probe light beam occurs inside the intracavity medium with strong absorption [15]. We note large negative GH shifts in the reflected probe light beam (solid line) and positive GH shifts in the transmitted light at certain incident angles $\theta$, as shown in Fig. 3(a).

The group indexes $\left(N_{g}\right)$ of the cavity corresponding to the reflected and transmitted probe light beams are calculated using Eq. (14). We find that the group index for the reflected beam is negative whenever the $\mathrm{GH}$ shift is negative. For example, at the incident angle $\theta=0.65 \mathrm{rad}$, where we observe large negative shift, the group index of the cavity is $N_{g}^{r}=-42.06$. The group index for the transmitted beam $N_{g}^{t}$ at the same incident angle is $=7.55$, which is positive and therefore positive GH shift is observed in the transmitted light beam. Here we emphasize that the negative GH shift appears only when the group index of the total cavity becomes negative. Similarly, the shift is positive only when the group index of the total cavity is positive. We also observe that the GH shift for the superluminal case is negative for certain choices of the incident angle and not for the whole range 0 to $\pi / 2 \mathrm{rad}$.

To see the effect of the driving field strength on the $\mathrm{GH}$ shift we change the driving field Rabi frequency from $\Omega_{d}=0$ to $\Omega_{d}=5 \Gamma$ while keeping the rest of the parameters unchanged. For this value of the driving field Rabi frequency the superluminal behavior of the light propagation switches to subluminal behavior. The corresponding GH shifts of the reflected and transmitted probe light beams for incident angle ranging from 0 to $\pi / 2$ rad are plotted in Fig. 3(b). We observe positive GH shifts with almost similar behavior in the reflected and transmitted light beams for all incident angles. The group index of the cavity in both cases also remains positive for each incident angle of the probe light. As an example, we calculate $N_{g}$ for the transmitted and reflected light beams when the incident angle is $\theta=0.65$ (rad). The group index $N_{g}$ is found to be 8.22 in both cases. Therefore, the GH shifts in the reflected and the transmitted probe light beams are positive for all incident angles and behave exactly similar. The inset of Fig. 3(b) shows the enlarged view of the GH shift for $\theta=0$ to $1 \mathrm{rad}$.

Next, we plot absolute values of the reflection and transmission coefficients in Figs. 4(a) and 4(b), respectively, versus incident angle $\theta$ of the probe light beam. The values of different parameters are the same as in Fig. 3(a). The plot shows dips in the reflection and transmission curves, which correspond to the angles satisfying the resonance conditions and where we observe large negative and positive shifts in the reflected and transmitted beams, respectively.

We have already noticed that the GH shift $S_{r, t}$ and the group index $N_{g}^{r, t}$ depend on the thickness of the cavity, $L$. Also, some earlier studies [11,12,35] show that the lateral shift behavior of the reflected and transmitted light is strongly influenced by the thickness of the medium. Therefore, we expect change in the behavior of the GH shifts in the reflected and transmitted light depending on thickness $d_{1}$ of the walls of the cavity and thickness $d_{2}$ of the intracavity medium. As an example, we reduce the thickness of the intracavity medium from $d_{2}=5 \mu \mathrm{m}$ to $d_{2}=1 \mu \mathrm{m}$ and plot the $\mathrm{GH}$ shifts in the reflected beam (solid line) and transmitted probe light beam (dashed line) versus incident angle $\theta$ again ranging from 0 to $\pi / 2 \mathrm{rad}$. We observe negative $\mathrm{GH}$ shifts in the reflected beam 

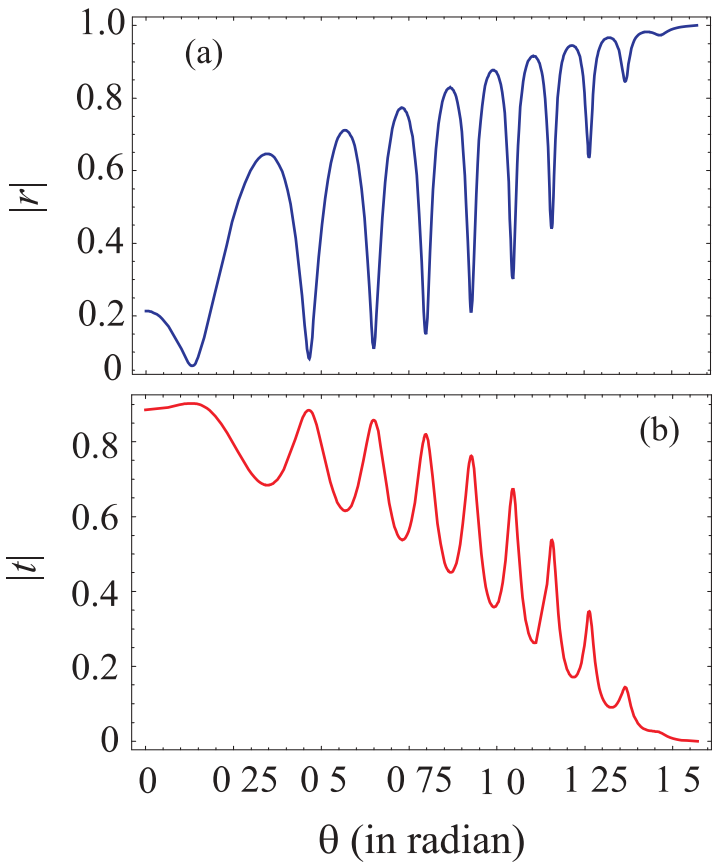

FIG. 4. (Color online) Three-level scheme showing (a) the absolute value of the reflection coefficient $|r|$ and (b) the absolute value of the transmission coefficient $|t|$ versus incident angle $\theta$. All the parameters are the same as in Fig. 3(a).

and positive $\mathrm{GH}$ shifts in the transmitted beam for $\Omega_{d}=0$ and positive shifts only in the reflected and transmitted beams when $\Omega_{d}=5 \gamma$ (see Fig. 5). This is similar to the earlier results in Fig. 3 when $d_{2}=5 \mu \mathrm{m}$; however, the number of oscillations is reduced with the decrease in the thickness of the intracavity
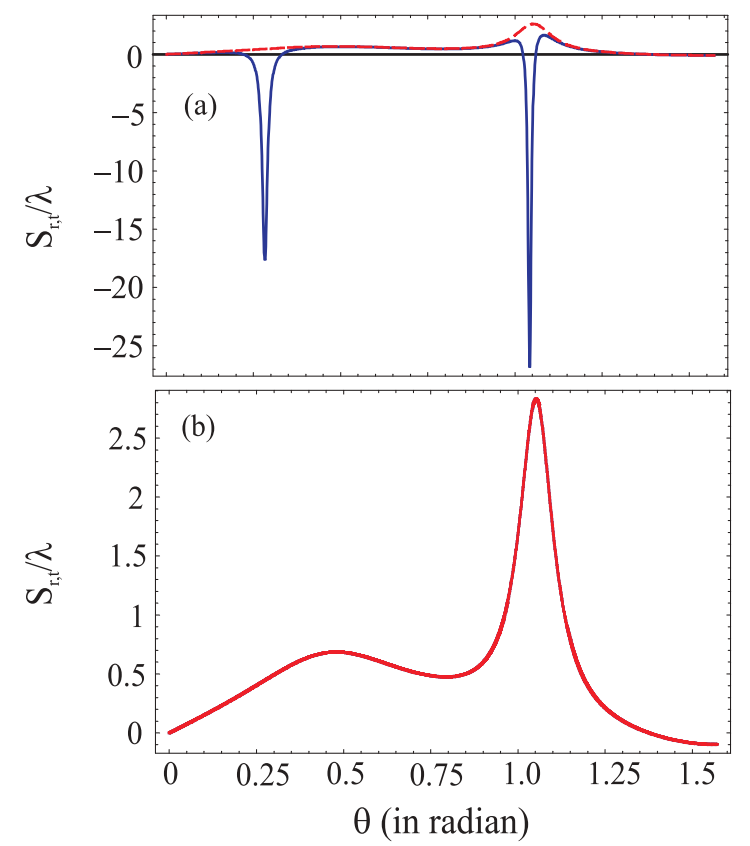

FIG. 5. (Color online) Three-level scheme showing the lateral shift in the reflected (solid line) and transmitted (dotted line) probe light beam incident at different angles ( 0 to $\pi / 2 \mathrm{rad}$ ) for (a) $\Omega_{d}=0$ and (b) $\Omega_{d}=5 \Gamma$. The other parameters are the same as in Fig. 3 except now $d_{2}=1 \mu \mathrm{m}$.

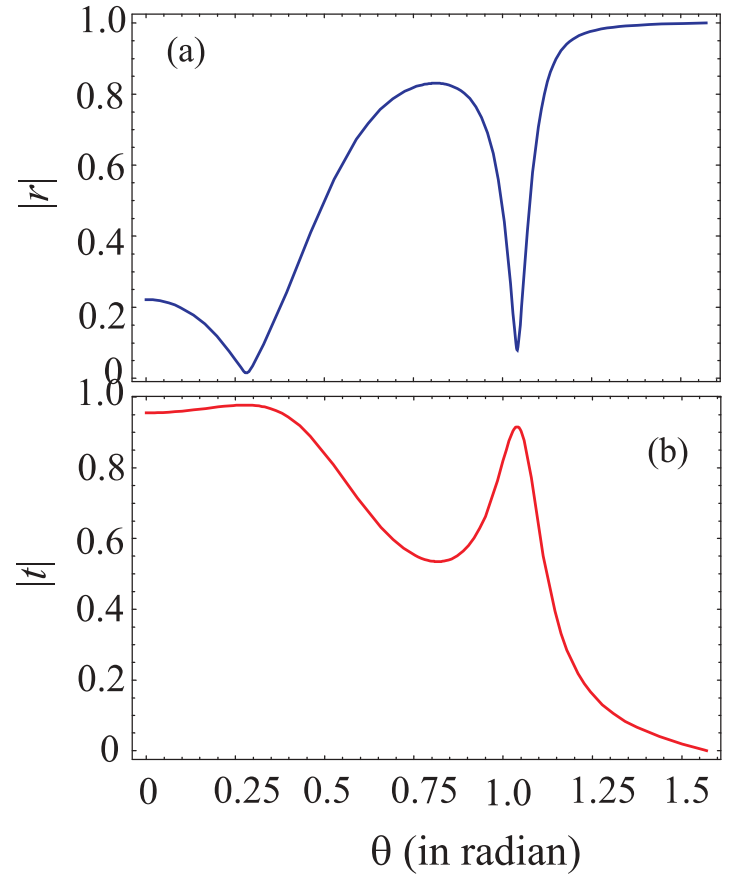

FIG. 6. (Color online) Three-level scheme showing (a) the absolute value of the reflection coefficient $|r|$ and (b) the absolute value of the transmission coefficient $|t|$ versus incident angle $\theta$. All the parameters are the same as in Fig. 5(a).

medium. The absolute values of the reflection and transmission coefficients are plotted in Figs. 6(a) and 6(b), respectively, which exhibit corresponding resonances where the large $\mathrm{GH}$ shifts appear.

As is mentioned earlier the absorption of the probe light beam inside the three-level EIT medium is very large for superluminal wave propagation. This is because when the driving field is switched off the three-level EIT system effectively reduces to a two-level atomic system where no dark states are present and the medium becomes a good absorber for the light beam passing through it. It has already been observed in [21] that superluminal propagation with reduced absorption of the probe light beam is possible in a four-level EIT system by using control of the coherent driving fields. Therefore, we now replace the intracavity medium of three-level atoms with four-level atoms. We again analyze the $\mathrm{GH}$ shift behavior in the reflected and transmitted beams for subluminal and superluminal light propagation through the cavity. The control knob here is the Rabi frequency $\Omega_{1}$, which corresponds to the atomic transition $|a\rangle$ to $|c\rangle$.

In Fig. 7, we show plots of the GH shift in the reflected (solid line) and transmitted (dotted line) probe light beams versus incident angle $\theta$ ranging from 0 to $\pi / 2$. The different parameters are chosen as $\Omega_{2}=\Omega_{3}=1.5 \gamma$ and $\gamma_{1}=\gamma_{2}=\gamma$. Initially, the control knob is set to be $\Omega_{1}=1.5 \gamma$, which correspond to subluminal light propagation through the intracavity medium of four-level atoms [21]. This is slow-light behavior and we observe positive GH shifts in both reflected and transmitted light beams for all incident angles $\theta$ ranging from 0 to $\pi / 2$ rad [see Fig. 7(a)]. The group index $N_{g}$ of the cavity in both reflected and transmitted light beam cases is also positive for each incident angle of the light beam. As an example, the group 

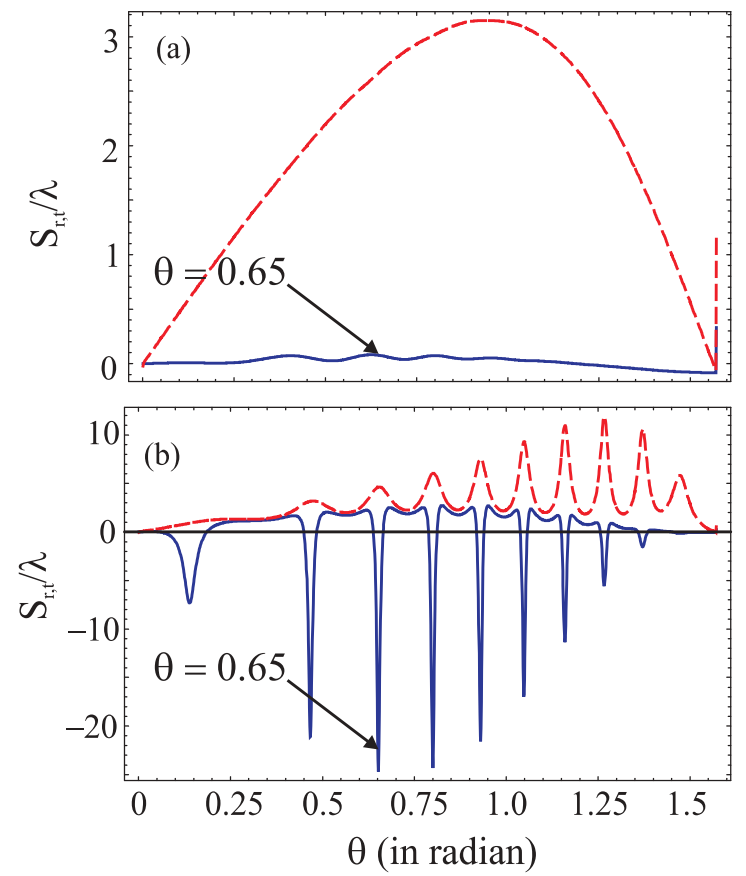

FIG. 7. (Color online) Four-level scheme showing GH shift in the reflected (solid line) and transmitted (dotted line) probe light beam incident at different incident angles ranging from 0 to $\pi / 2 \mathrm{rad}$ with $\Omega_{2}=\Omega_{3}=1.5 \gamma, \gamma_{1}=\gamma_{2}=\beta=\gamma$, and $\omega_{a d} /(2 \pi)=300 \mathrm{THz}$. (a) $\Omega_{1}=1.5 \gamma$. (b) $\Omega_{1}=20 \gamma$. The other parameters are the same as in Fig. 3.

index of the cavity for the reflected and transmitted light at the incident angle $\theta=0.65 \mathrm{rad}$ are calculated using Eq. (14). The group index for the reflected beam is found to be 0.278 and for the transmitted beam it is equal to 5.996 .

Next, we change the controlling parameter $\Omega_{1}$ and choose $\Omega_{1}=20 \gamma$ while the other parameters are unchanged. This now corresponds to superluminal propagation of the probe light beam with a considerably reduced absorption [21]. Figure 7(b) shows large negative GH shifts in the reflected light (solid line) at certain incident angles of the probe light, whereas the transmitted light (dashed line) undergoes positive $\mathrm{GH}$ shifts. The corresponding group index for negative shift is negative and for positive shift is positive. For example, the group index of the cavity at the incident angle $\theta=0.65 \mathrm{rad}$ is equal to -36.99 for the reflected light beam and 7.514 for the transmitted light beam. In Figs. 8(a) and 8(b), we plot the absolute values of the reflection and transmission coefficients versus incident angle $\theta$ for the case of superluminal wave propagation only (i.e., when $\Omega_{1}=20 \gamma$ ). The plots show a similar behavior to that observed in Fig. 4 for the three-level atomic configuration. The dips in the reflection and transmission coefficients again correspond to the resonance conditions where we observe large negative and positive $\mathrm{GH}$ shifts. Finally, in Fig. 9, we show the dependence of the cavity group index $N_{g}^{r}$ and the GH shift $S_{r} / \lambda$ on the control knob values. For a three-level intracavity medium the control knob is the driving field Rabi frequency $\Omega_{d}$ and for a four-level atomic system the driving field Rabi frequency $\Omega_{1}$ is the controlling parameter. In Fig. 9(a), we plot $N_{g}^{r}$ versus $\Omega_{1} / \gamma$, which ranges from 1.5 to 30 , for a four-level atomic system (main plot). The

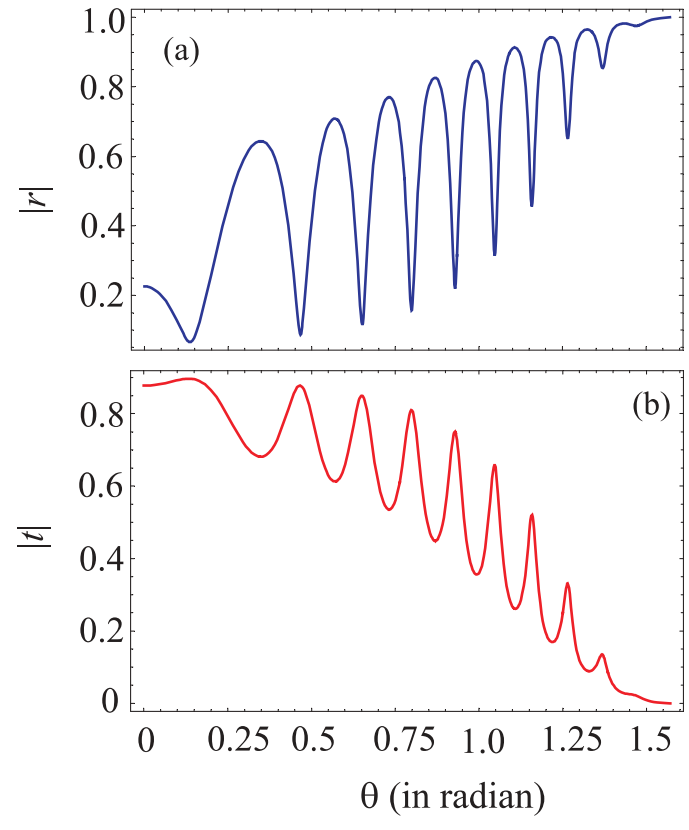

FIG. 8. (Color online) Four-level scheme showing (a) the absolute value of the reflection coefficient $|r|$ and (b) the absolute value of the transmission coefficient $|t|$ versus incident angle $\theta$. All the parameters are the same as in Fig. 7(b).

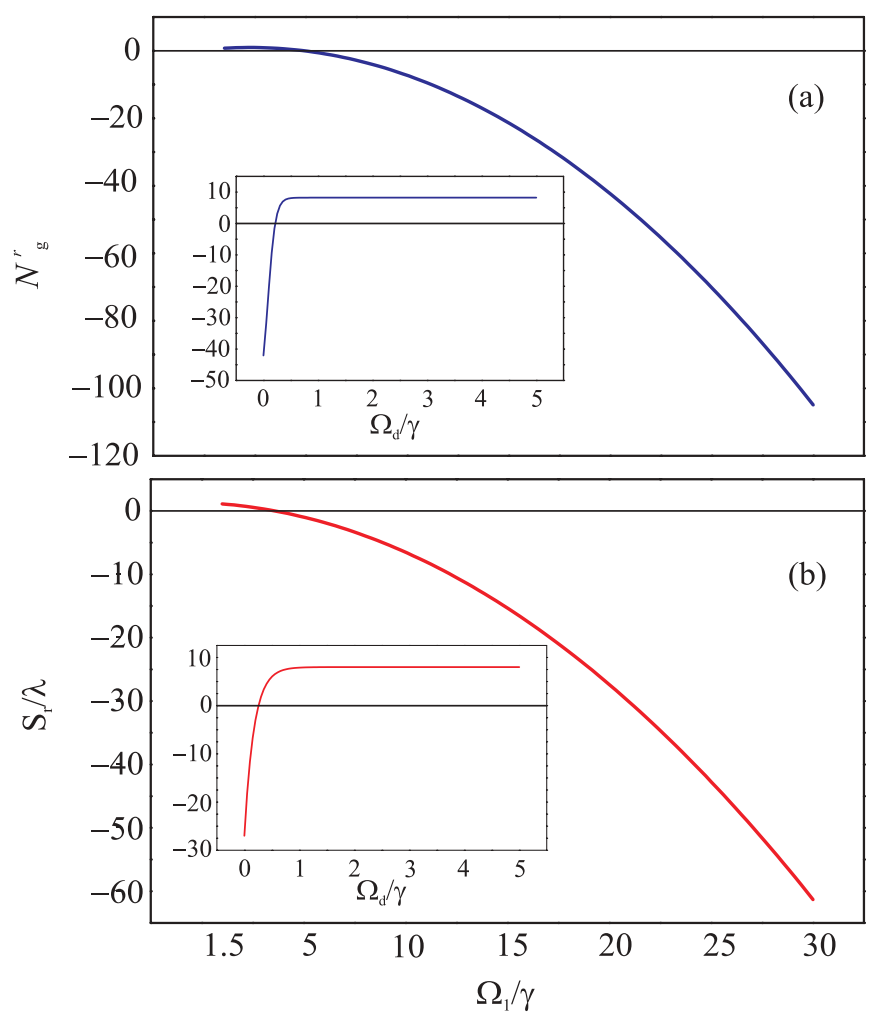

FIG. 9. (Color online) (a) The group index of the cavity versus the control knob for a four-level atomic system (main plot) and a three-level atomic system (inset). (b) The GH shift in the reflected beam versus the control knob for a four-level atomic system (main plot) and a three-level atomic system (inset). The incident angle is $\theta=0.65 \mathrm{rad}$ and the rest of the parameters are the same as in Figs. 3(a) and 7(b) for three-level and four-level systems, respectively. 
incident angle of the probe light beam is $\theta=0.65 \mathrm{rad}$ while the other parameters are the same as in the case of Fig. 7(b). We observe that the cavity group index $N_{g}^{r}$ is positive for the range 1.5 to almost 4 of $\Omega_{1} / \gamma$. The corresponding GH shift of the reflected beam is also positive in that range of $\Omega_{1} / \gamma$ [see Fig. 9(b), main plot]. However, $N_{g}^{r}$ becomes negative when $\Omega_{1} / \gamma$ gets a value higher than 4 and remains negative for any further higher value of the control knob. The corresponding GH shift in the reflected beam is also negative. This is in agreement with our earlier analysis that whenever the cavity group index is negative the GH shift is also negative. The insets in Figs. 9(a) and 9(b) show the dependence of the cavity group index $N_{g}^{r}$ and GH shift $S_{r} / \lambda$ on the driving field Rabi frequency $\Omega_{d} / \gamma$ in the three-level atomic system. This is again in agreement with our earlier observations in Fig. 3(a).

\section{CONCLUSION}

We have used three-level and four-level atomic systems with EIT configuration to observe changes in the GH shift behavior of the reflected and transmitted light beams. We have considered resonant interactions of the driving and probe fields with the corresponding atomic transitions. The probe light is incident on the cavity at different angles ranging from 0 to $\pi / 2$ rad. We have observed negative and positive GH shifts in the reflected beam, corresponding to superluminal and subluminal propagation of the probe light beam, respectively, provided that the group index of the cavity is negative for the former case and positive for the latter. The transmitted beam, however, exhibits only positive GH shifts in superluminal as well as subluminal wave propagation. This implies that the behavior of the GH shifts basically depends on the group index of the cavity.

We have observed that the group index of the cavity is always positive when the intracavity medium is transparent for the incident probe light beam (subluminal wave propagation). Therefore, the GH shift is also positive for incident angles ranging from 0 to $\pi / 2 \mathrm{rad}$ in the subluminal regime of the intracavity medium. On the other hand when the characteristics of the intracavity medium changes to superluminal behavior we get a different picture. In this case the group index of the cavity is negative as well as positive for the reflected beam depending on the incident angle and thickness of the cavity. For certain incident angles, we have observed large negative GH shifts in the reflected probe light beam, which correspond to the negative group index of the cavity. We have also noticed that the four-level EIT medium is more suitable for observing negative GH shifts. This is because the absorption of the probe light beam is considerably reduced in the four-level EIT medium. From this analysis we may conclude that switching of the GH shifts from positive to negative and vice versa is possible via coherent control of the driving field in a threeor four-level intracavity medium with a reduced absorption of the light during propagation in the latter configuration.

A possible real, physical three-level EIT system may be considered as a cavity consisteing of $\mathrm{Sr}$ [22] or $\mathrm{Pb}$ [23] atoms with narrow-band pulsed laser radiation. The observation of EIT in the experiment using Sr atoms [22] is based on a similar three-level $\Lambda$ configuration as is mentioned in Fig. 2(a). In the recent past, some experimental observations of EIT in fourlevel atomic systems has also been reported (e.g., an inverted-Y configuration [36], photon switching by quantum interference [37], coherence switching [38], doubly dressed states in cold atoms [39], giant Kerr nonlinearities via EIT [40], and narrow absorptive resonances [41]) and the double dark scheme of four-level atomic coherence has been utilized to obtain strong laser gain [42].

However, most of the experimental studies on EIT have been carried out in gases, but there are few schemes where EIT has been observed in solids as well. In 1995, the possibility of creating dark states in semiconductor such as zinc-blende structure (GaAs) was discussed [24]. This work has demonstrated that the fundamental three-level coherence effects related to the dark states in atomic system have analogies in semiconductor systems. Later, in 1997 and 1998 reports on the observation of EIT in ruby [25] and in $\operatorname{Pr}^{3+}: \mathrm{Y}_{2} \mathrm{SiO}_{5}[26,27]$ have been published. Further, a few experiments have been suggested for observation of EIT in semiconductors which are based on $\mathrm{Cu}_{2} \mathrm{O}$ [28] and $\mathrm{CuCl}$ [29].

We again mention here that in the analysis we have considered a well-collimated probe light beam with a narrow angular spectrum, $\Delta k \ll k$, and the lateral shift is calculated using stationary phase theory. This means that our results are based on stationary phase theory. However, it has already been shown in the earlier work [18] that these results are still valid in the real system when the incident probe light beam has a finite width (typically, with a Gaussian profile).

\section{ACKNOWLEDGMENTS}

We thank COMSTECH for support. The authors are grateful to Li-Gang Wang for some helpful discussions. The research work of M.S.Z is supported by a grant from the Qatar National Research Fund (QNRF).
[1] F. Goos and H. Hanchen, Ann. Phys. 1, 333 (1947).

[2] R. H. Renard, J. Opt. Soc. Am. 54, 1190 (1964).

[3] H. K. V. Lotsch, J. Opt. Soc. Am. 58, 551 (1968).

[4] T. Tamir and H. L. Bertoni, J. Opt. Soc. Am. 61, 1397 (1971).

[5] H. M. Lai and S. W. Chan, Opt. Lett. 27, 680 (2002).

[6] W. J. Wild and C. L. Giles, Phys. Rev. A 25, 2099 (1982).

[7] J. L. Birman, D. N. Pattanayak, and A. Puri, Phys. Rev. Lett. 50, 1664 (1983).
[8] E. Pfleghaar, A. Marseille, and A. Weis, Phys. Rev. Lett. 70, 2281 (1993).

[9] H. M. Lai and S. W. Chan, Opt. Lett. 27, 680 (2002).

[10] P. R. Berman, Phys. Rev. E 66, 067603 (2002).

[11] C.-F. Li, Phys. Rev. Lett. 91, 133903 (2003).

[12] X. Chen and C.-F. Li, Phys. Rev. E 69, 066617 (2004).

[13] T. Hashimoto and T. Yoshino, Opt. Lett. 14, 913 (1989). 
[14] N. J. Harrick, Phys. Rev. Lett. 4, 224 (1960).

[15] M. O. Scully and M. S. Zubairy, Quantum Optics (Cambridge University Press, Cambridge, 1997).

[16] M. O. Scully, Phys. Rev. Lett. 67, 1855 (1991).

[17] M. O. Scully, Phys. Rev. Lett. 67, 1855 (1991); M. Fleischhauer, C. H. Keitel, M. O. Scully, C. Su, B. T. Ulrich, and S.-Y. Zhu, Phys. Rev. A 46, 1468 (1992).

[18] L. G. Wang, M. Ikram, and M. S. Zubairy, Phys. Rev. A 77, 023811 (2008).

[19] M. Fleischhauer, C. H. Keitel, M. O. Scully, and C. Su, Opt. Commun. 87, 109 (1992); M. O. Scully and S.-Y. Zhu, ibid. 87, 134 (1992); S. Sultana and M. S. Zubairy, Phys. Rev. A 49, 438 (1994); O. Kocharovskaya, P. Mandel, and M. O. Scully, Phys. Rev. Lett. 74, 2451 (1995); A. S. Zibrov, M. D. Lukin, L. Hollberg, D. E. Nikonov, M. O. Scully, H. G. Robinson, and V. L. Velichansky, ibid. 76, 3935 (1996); S. E. Harris, Phys. Today 50, 36 (1997).

[20] T. Quang and H. Freedhoff, Phys. Rev. A 48, 3216 (1993).

[21] M. Sahrai, H. Tajalli, K. T. Kapale, and M. Suhail Zubairy, Phys. Rev. A 70, 023813 (2004).

[22] K.-J. Boller, A. Imamoglu, and S. E. Harris, Phys. Rev. Lett. 66, 2593 (1991).

[23] J. E. Field, K. H. Hahn, and S. E. Harris, Phys. Rev. Lett. 67, 3062 (1991).

[24] M. Lindberg and R. Binder, Phys. Rev. Lett. 75, 1403 (1995).

[25] Y. Zhao, C. Wu, B.-S. Ham, M. K. Kim, and E. Awad, Phys. Rev. Lett. 79, 641 (1997).

[26] B. S. Ham, M. S. Shahriar, and P. R. Hemmer, Opt. Lett. 22, 1138 (1997).

[27] K. Ichimura, K. Yamamoto, and N. Gemma, Phys. Rev. A 58, 4116 (1998).
[28] M. Artoni, G. C. L. Rocca, and F. Bassani, Europhys. Lett. 49, 445 (2000).

[29] S. Chesi, M. Artoni, G. C. La Rocca, F. Bassani, and A. Mysyrowicz, Phys. Rev. Lett. 91, 057402 (2003).

[30] K. Artmann, Ann. Phys. 2, 87 (1948).

[31] M. Born and E. Wolf, Principles of Optics (Cambridge University Press, Cambridge, 1999).

[32] N. H. Liu, S.-Y. Zhu, H. Chen, and X. Wu, Phys. Rev. E 65, 046607 (2002).

[33] G. S. Agarwal and S. Dasgupta, Phys. Rev. A 70, 023802 (2004); L. G. Wang, S. Qamar, S.-Y. Zhu, and M. S. Zubairy, ibid. 77, 033833 (2008).

[34] E. Döpel, J. Mod. Opt. 37, 237 (1990); O. F. Siddiqui, M. Mojahedi, and G. V. Eleftheriades, IEEE Trans. Antennas Propag. 51, 2619 (2003).

[35] L. G. Wang, H. Chen, and S.-Y. Zhu, Opt. Lett. 30, 2936 (2005); M. Cheng, Y. Zhou, Y. Li, and X. Li, J. Opt. Soc. Am. B 25, 773 (2008).

[36] A. Joshi and M. Xiao, Phys. Lett. A317, 370 (2003); A. Joshi, Phys. Rev. B 79, 115315 (2009).

[37] S. E. Harris and Y. Yamamoto, Phys. Rev. Lett. 81, 3611 (1998).

[38] B. S. Ham and P. R. Hemmer, Phys. Rev. Lett. 84, 4080 (2000).

[39] M. Yan, E. G. Rickey, and Y. Zhu, Phys. Rev. A 64, 013412 (2001).

[40] H. Schmidt and A. Imamoglu, Opt. Lett. 21, 1936 (1996).

[41] M. G. Bason, A. K. Mohapatra, K. J. Weatherill, and C. S. Adams, J. Phys. B 42, 075503 (2009).

[42] E. S. Fry, M. D. Lukin, T. Walther, and G. R. Welch, Opt. Commun. 179, 499 (2000). 